\title{
Structure and Energy of Crystal Interfaces I. Formal Development
}

\author{
By.N. H. Fletcher $\dagger$ and Peggy L. Adamson $\ddagger$ \\ Department of Physics, University of New England, \\ Armidale, N.S.W., Australia
}

[Received 19 March 1966]

\begin{abstract}
A variational method is developed for calculating the energy of an interface between two crystals of arbitrary structures and relative orientations. The method takes account of details of the interaction potential between atoms and allows for all possible displacements of atoms near the interface but, by considering the Fourier transforms of all the quantities involved, the number of significant variational parameters is kept small. The form of the variational function is such that deductions can be made about the existence of cusped energy minima for certain simple matchings between the two crystal lattices. The formalism contains the dislocation model of a crystal boundary as a limiting case but also allows for calculations in more general situations.
\end{abstract}

\section{§ 1. INTRODUCTION}

A knowledge of the energy and structure of an interface between two crystalline solids is of importance in many branches of crystal physics and much experimental and theoretical work has been directed towards increasing our understanding of the subject (for reviews, see Brooks 1952, Read 1953, Amelinckx and Dekeyser 1959). Theoretical studies have generally been based upon some sort of dislocation model for the interface (Frank and van der Merwe 1949, van der Merwe 1950, 1963), though it has recently become marginally feasible, with modern computers, to consider the displacements of individual atoms near boundaries or other imperfections of simple form.

In an earlier paper (Fletcher 1964), one of the present authors proposed a simple variational technique for calculating the energy of an interface between two crystals of different structure and arbitrary orientation, the approximation of the method being intermediate between that of a dislocation treatment and a rigorous atom-by-atom calculation. By applying this method to a one-dimensional interface it was demonstrated that, whilst the treatment could be reduced to a dislocation model for small misfits between the two lattices, it was much better adapted than was the dislocation model to dealing with large misfits and showed up the existence of cusped energy minima for misfit ratios like $2: 1$ or $3: 2$. These

† On leave at H. H. Wills Physics Laboratory, University of Bristol.

$\ddagger$ Present address: Department of Physics, University of Zambia, Lusaka, Zambia. 
cusped minima had previously been discussed, for the case of a simple tilt boundary, by Read and Shockley (1950) and their existence has since also been demonstrated, using a generalized hierarchical dislocation model, by du Plessis and van der Merwe (1965).

The variational method previouslý used, howevier, suffers from ànumber of approximations and inconsistencies which stem from the procedure of treating the two crystals separately and then superposing the results. It also neglects displacements normal to the interface which, in any real crystal, are likely to play a considerable part in determining the interface structure and energy. The purpose of the present paper is to present the formal theory of the variational method in a generalized form which removes these inconsistencies and approximations but which is still realistic from the point of view of actual numerical calculation. In a subsequent publication we shall present the results of such an explicit calculation for a real two-dimensional interface and discuss how the conclusions can be of assistance in understanding certain phenomena connected with crystal nucleation and epitaxial growth.

\section{§ 2. The Variational Method}

The quantity which we wish to evaluate is the energy of the interface between two low-index faces of different crystals placed in intimate contact, with arbitrary relative orientation. For convenience we take the sum of the surface energies of the two separated crystals as a zero.

It is clear that the atoms near the interface will be displaced elastically away from their normal positions in the lattice so that there will be a considerable positive contribution from elastic strain energy in the body of each crystal in addition to the negative contribution made by the pairby-pair atomic interactions across the interface. We shall restrict our attention in what follows to cases where the adhesive energy can be considered as made up of such short-range pair-wise interactions, so that the discussion may not be directly applicable to metallic interfaces where conduction electrons play a large part in the binding energy.

In a detailed 'brute-force' computer solution one could vary the positions of each atom in the bi-crystal until the energy had been minimized but, even in simple situations, the number of variational parameters required is of the order of $10^{4}$ so that a great deal of computer time is required and no general pattern emerges until a great number of specific cases have been studied.

The basis of the present method is to observe that, if the displacements of atoms immediately adjacent to the interface are analysed in a Fourier series, certain resonance terms in this series are then of preponderant importance. The amplitudes and polarizations of these Fourier components then provide a small set of variational parameters with respect to which the energy may be minimized. As for the energy component due to elastic strain, the crystals may validly be approximated by semi-infinite elastic continua and the elastic problem is then one which has been studied 
in detail elsewhere. The total number of variational parameters requirêd to treat a general interface is typically less than 100 and, because the variational expression has a transparent analytical form, the most significant parameters may easily be selected to reduce the effective member still further. Again, because of the form of the variational expression, the minimization programme can be written in a form which will converge quickly and, however many parameters are arbitrarily set equal tọ zero, the method will always yield a valid upper bound to the energy. .

In the discussion to follow we will calculate first the pair-wise interaction energy across the boundary and then the elastic strain energy. Finally we combine these two components and discuss the form of the variational expression for the total energy.

\section{§ 3. INTERACTION ENERGY}

Suppose that one semi-infinite crystal, which we shall call the substrate, consists of atoms of type A and that the other crystal, the overgrowth, is of atoms of type B. Then the whole of the interaction energy must be expressible in terms of the interaction potential $v_{\mathrm{AB}}(r)$ between an $\mathrm{A}$ and a B atom separated by a distance $r$. We shall consider only this simplest case in which each crystal is monatomic but the argument can easily be extended to more general cases. We shall also assume the range of $v_{\mathrm{AB}}(r)$ to be so short that only nearest neighbours (or at most next-nearest neighbours) need to be considered in any interaction.

Our first task is to calculate the potential felt by a B atom near the complete A substrate when it is subject to a set of arbitrary distortions. We can then sum over the atoms of a whole arbitrarily distorted B crystal to find the interaction energy in this general state.

\subsection{Substrate Potential}

Consider first the potential $V_{0}$ experienced by a B atom just outside a low-index surface of a perfect, undistorted substrate of $\mathbf{A}$ atoms whose positions are given by the vectors $\mathbf{R}$. If the $B$ atom is at the point $\mathbf{r}$ then

$$
V_{0}(\mathbf{r})=\sum_{\mathbf{R}} v_{A B}(|\mathbf{r}-\mathbf{R}|) \equiv \sum_{\mathbf{R}} v(\mathbf{r}-\mathbf{R})
$$

where the second form of writing shows the simplified notation we shall use henceforth. Because of the assumed short range of $v(\mathbf{r})$, the only effective terms in the $\mathbf{R}$ summation are those applying to atoms in the surface layer (nearest neighbours) or the layer immediately below this.

This potential can be resolved into Fourier components $V_{0}(\mathbf{k})$, given by:

$$
\begin{aligned}
& \nabla_{0}(\mathbf{k})=\Omega^{-i} \int \exp (-i \mathbf{k} \cdot \mathbf{r}) \sum_{\mathbf{R}} v(\mathbf{r}-\mathbf{R}) d \mathbf{r} \\
& =\sum_{\mathbf{R}} \exp (-i \mathbf{k} \cdot \mathbf{R}) \Omega^{-1} \int \exp [-i \mathbf{k} \cdot(\mathbf{r}-\mathbf{R})] v(\mathbf{r}-\mathbf{R}) d(\mathbf{r}-\mathbf{R}) \\
& =\sum_{\mathbf{R}} \exp (-i \mathbf{k} \cdot \mathbf{R}) v(\mathbf{k}), \quad \cdot \cdot \cdot \cdot \cdot \cdot \cdot \cdot \cdot \cdot \cdot \cdot \cdot \cdot
\end{aligned}
$$

where $v(\mathbf{k})$ is the Fourier transform of the atomic interaction potential 
$v(\mathbf{r})$ and $\Omega$ is the volume over which the integration is performed. Since $v(\mathbf{r})$ is real, we have:

$$
v(-\mathbf{k})=v(\mathbf{k})^{*}
$$

We now note that, because the sum over $\mathbf{R}$ in (2) is only a half-space, we cannot immediately write it as $\delta_{\mathbf{k}} \mathscr{G}$, where $\mathscr{G}$ are the reciprocal lattice vectors of the substrate. However, if $\mathbf{k}_{1}$ is the component of $\mathbf{k}$ parallel to the interface and $g$ are the components of $\mathscr{E}$ in this same plane, we can write:

$$
\lim _{N \rightarrow \infty} \sum_{\mathbf{R}} \exp \left(-i \mathbf{k}_{1}, \mathbf{R}\right)=N \delta_{\mathbf{k}_{1}, \mathbf{g}}, \quad . \quad \text {. . . . . . }
$$

where $N$ is the number of lattice points $\mathbf{R}$ in the summation.

Strictly speaking we now require a continuous range of components of $\mathbf{k}$ normal to the interface but, because the interatomic potentials are of short range, we can confine our attention to a region of thickness $h$ adjacent to the surface and imagine the potential distribution in a symmetric slab of thickness $2 h$ to be repeated periodically outside our region of interest. The total potential can then be described by giving Fourier components at all points of a reciprocal lattice with vectors:

$$
\mathbf{G} \equiv \mathbf{g}+2 \pi n \hat{\mathbf{n}} / 2 h ; \quad(n=0, \pm 1, \ldots)
$$

where $\hat{\mathbf{n}}$ is the unit vector normal to the surface. Equation (4) can then be generalized to read:

$$
\lim _{N \rightarrow \infty} \sum_{\mathbf{R}} \exp (-i \mathbf{k} \cdot \mathbf{R})=N \delta_{\mathbf{k}, \mathbf{G}} \cdot \ldots . . .
$$

$\mathbf{G}$ is not, of course, in general coincident with the ordinary reciprocal lattice $\mathscr{G}$, except in the plane $n=0$.

We now introduce distortions into the substrate. To make it easier to count modes we first bring the complete B crystal up to the substrate and note that by a pure displacement combined with an arbitrarily small change in the lattice parameters of one of the crystals it will always be possible to bring some three atoms of the $\mathrm{B}$ crystal into coincidence with three atoms of the A crystal. The two vectors in the surface joining one of these atoms to the other two then define a plane superlattice in which the behaviour of any distortions must be periodic. The size of this superlattice will generally be very large and can be let tend to infinity so that it places no physical restrictions on the problem. At the same time, by reducing the number of distortion components to a denumerable infinity, it effects a considerable algebraic simplification.

Because of the existence of this superlattice we can now decompose a general distortion of the surface of the substrate in which the atom at $\mathbf{R}$ moves to $\mathbf{R}+\mathbf{F}(\mathbf{R})$ as :

$$
\mathbf{F}(\mathbf{R})=-\sum_{\mathbf{K}} \exp (i \mathbf{K} \cdot \mathbf{R}) \mathbf{F}_{\mathbf{K}}
$$

where $\mathbf{K}$ are vectors of the reciprocal lattice of the superlattice. These vectors $\mathbf{K}$ are always parallel to the interface but the vector components $\mathbf{F}(\mathbf{K})$ may have any orientation. Because only atoms in the surface of the substrate effectively contribute to the potential, we may neglect the (exponential) variation of $\mathbf{F}$ with depth below the surface. 
Since $\mathbf{F}(\mathbf{R})$ is real,

and we can write:

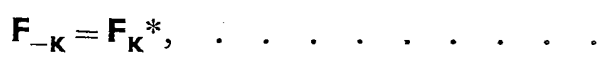

$$
\mathbf{F}_{\mathbf{K}} \equiv \mathbf{C}_{\mathbf{K}}-i \mathbf{D}_{\mathbf{k}}
$$

where

$$
\mathbf{C}_{-\mathrm{K}}=\mathbf{C}_{\mathrm{K}} ; \quad \mathbf{D}_{-\mathrm{K}}=-\mathbf{D}_{\mathrm{K}} \cdot \quad \cdot \quad \cdot \quad . \quad . \quad .
$$

Equation (7) then becomes:

$$
\mathbf{F}(\mathbf{R})=-\Sigma^{+} 2\left[\mathbf{D}_{\mathbf{K}} \cdot \sin (\mathbf{K} \cdot \mathbf{R})+\mathbf{C}_{\mathbf{K}} \cos (\mathbf{K} \cdot \mathbf{R})\right]-\mathbf{C}_{\mathbf{0}}, \quad \cdot \quad \cdot(11)
$$

where the + on the summation indicates that it extends over only half of $\mathbf{K}$-space, and we have explicitly included the $\mathbf{K}=0$ term, representing a shift in origin.

Substitution of $\mathbf{R} \rightarrow \mathbf{R}+\mathbf{F}(\mathbf{R})$ into (2) then gives general potential eomponents for the distorted substrate:

$$
\begin{aligned}
V(\mathbf{k})=v(\mathbf{k}) \sum_{\mathbf{R}} \exp \left[-i \mathbf{k} \cdot\left(\mathbf{R}-\mathbf{C}_{0}\right)\right] \prod_{\mathbf{K}} \prod^{+} & \left\{\exp \left[2 i \mathbf{k} \cdot \mathbf{D}_{\mathbf{K}} \sin (\mathbf{K} \cdot \mathbf{R})\right]\right. \\
& \left.\times \exp \left[2 i \mathbf{k} \cdot \mathbf{C}_{\mathbf{K}} \cos (\mathbf{K} \cdot \mathbf{R})\right]\right\} .
\end{aligned}
$$

To reduce this we make use of the Bessel function expansion (Watson 1944) :

$$
\exp \left\{\frac{x}{2}\left(t-\frac{1}{t}\right)\right\}=\sum_{n=-\infty}^{\infty} J_{n}(x) t^{n}
$$

with $t=\exp (i \mathbf{K} \cdot \mathbf{R}), \quad x=2 \mathbf{k} \cdot \mathbf{D}_{\mathbf{K}}$ and $t=\exp [i \mathbf{K} \cdot \mathbf{R}+i(\pi / 2)], \quad x=2 \mathbf{k} \cdot \mathbf{C}_{\mathbf{K}}$ respectively, giving:

$$
\begin{aligned}
V(\mathbf{k}) & =v(\mathbf{k}) \sum_{\mathbf{R}} \exp \left[-i \mathbf{k} \cdot\left(\mathbf{R}-\mathbf{C}_{\mathbf{0}}\right)\right] \\
& \times \prod_{\mathbf{K}}^{+}\left[\sum_{n, m=-\infty}^{\infty} i^{m} J_{n}\left(2 \mathbf{k} \cdot \mathbf{D}_{\mathbf{k}}\right) J_{m}\left(2 \mathbf{k} \cdot \mathbf{C}_{\mathbf{k}}\right) \exp [i(n+m) \mathbf{K} \cdot \mathbf{R}]\right] .
\end{aligned}
$$

We can now use the relation (6) to pick out the allowed terms in this expansion, giving:

$$
\begin{aligned}
V(\mathbf{k})=N v(\mathbf{k}) \exp \left(i \mathbf{k} \cdot \mathbf{C}_{0}\right)\left\{\Pi^{+} J_{0}\left(2 \mathbf{k} \cdot \mathbf{D}_{\mathbf{k}}\right) J_{0}\left(2 \mathbf{k} \cdot \mathbf{C}_{\mathbf{k}}\right)\right\} \\
\left\{\delta_{\mathbf{k}, \mathbf{G}}+\sum_{n=1}^{\infty} \sum_{\mathbf{K}}\left[\frac{J_{n}\left(2 \mathbf{k} \cdot \mathbf{D}_{\mathbf{K}}\right)}{J_{0}\left(2 \mathbf{k} \cdot \mathbf{D}_{\mathbf{K}}\right)}+i^{n} \frac{J_{n}\left(2 \mathbf{k} \cdot \mathbf{C}_{\mathbf{K}}\right)}{J_{0}\left(2 \mathbf{k} \cdot \mathbf{C}_{\mathbf{k}}\right)}\right]_{1} \delta_{\mathbf{k}-n \mathbf{K}, \mathbf{G}}\right. \\
+\sum_{n, m=1}^{\infty} \sum_{\mathbf{K}, \mathbf{L}}\left[\frac{J_{n}\left(2 \mathbf{k} \cdot \mathbf{D}_{\mathbf{K}}\right) J_{m}\left(2 \mathbf{k} \cdot \mathbf{D}_{\mathbf{L}}\right)}{J_{0}\left(2 \mathbf{k} \cdot \mathbf{D}_{\mathbf{K}}\right) J_{0}\left(2 \mathbf{k} \cdot \mathbf{D}_{\mathbf{L}}\right)}+i^{m} \frac{J_{n}\left(2 \mathbf{k} \cdot \mathbf{D}_{\mathbf{K}}\right) J_{m}\left(2 \mathbf{k} \cdot \mathbf{C}_{\mathbf{L}}\right)}{J_{0}\left(2 \mathbf{k} \cdot \mathbf{D}_{\mathbf{K}}\right) J_{0}\left(2 \mathbf{k} \cdot \mathbf{C}_{\mathbf{L}}\right)}\right. \\
\left.\left.+i^{n+m} \frac{J_{n}\left(2 \mathbf{k} \cdot \mathbf{C}_{\mathbf{K}}\right) J_{m}\left(2 \mathbf{k} \cdot \mathbf{C}_{\mathbf{L}}\right)}{J_{0}\left(2 \mathbf{k}, \mathbf{C}_{\mathbf{K}}\right) J_{0}\left(2 \mathbf{k} \cdot \mathbf{C}_{\mathbf{L}}\right)}\right]_{2} \delta_{\mathbf{K}-n \mathbf{k}-m \mathbf{L}, \mathbf{G}}+\ldots\right\}
\end{aligned}
$$

where we have used the freedom of sign of the order of the Bessel functions to extend the $\mathbf{K}, \mathbf{L} \ldots$...summations to the whole space. The truncated terms in (15) involve products of three or more Bessel functions of nonzero order and are easily written down if required. The numbers on the brackets will be used to refer to them later.

We note that the potential of the distorted lattice, as well as containing the Fourier components of the undistorted potential with reduced strength, now has satellite components around each of them, corresponding to wavenumber shifts produced by the distortions. The analysis is, in fact, a 
generalized form of that which might be employed in discussing the frequency or phase modulation of a radio frequency carrier wave in communication theory.

\subsection{Interaction Energy}

Now that we have calculated the interaction potential within a region of thickness $h$ outside the substrate surface, it is a relatively simple matter, using the same techniques, to add up the contributions of all atomic positions in the overgrowth to obtain the interaction energy. Because of the short range of $v(r)$ this sum has its major contribution from the surface layer of atoms in the overgrowth and, to simplify the algebra; we will omit any contribution from the next-nearest layer.

If the atomic positions in the undistorted overgrowth are denoted by $\mathbf{R}^{\prime}$ and the distortions by $\mathbf{F}^{\prime}\left(\mathbf{R}^{\prime}\right)$ then these may, because of the existence of the superlattice, be analysed in terms of Fourier components based upon the same set.of vectors $\mathbf{K}$ as used for the substrate, to give:

$$
\mathbf{F}^{\prime}\left(\mathbf{R}^{\prime}\right)=\sum_{\mathbf{K}}{ }^{+} 2\left[\mathbf{D}_{\mathbf{K}}{ }^{\prime} \sin \left(\mathbf{K} \cdot \mathbf{R}^{\prime}\right)+\mathbf{C}_{\mathbf{K}}{ }^{\prime} \cos \left(\mathbf{K} \cdot \mathbf{R}^{\prime}\right)\right]+\mathbf{C}_{\mathbf{0}}^{\prime} \cdot .
$$

The change in sign, as compared with (11), is for reasons of symmetry.

The total interaction energy can then be written:

$$
E_{i}^{\prime}=\frac{1}{N^{\prime} A^{\prime}} \sum_{\mathbf{R}^{\prime}} \sum_{\mathbf{k}} V(\mathbf{k}) \exp \left(i \mathbf{k} \cdot\left[\mathbf{R}^{\prime}+\mathbf{F}^{\prime}\left(\mathbf{R}^{\prime}\right)\right]\right), \quad \text {, . }
$$

where $N^{\prime}$ is the number of atoms in the sum and $A^{\prime}$ is the surface area per atom.

The expanded form obtained by substituting. (16) in (17) may be evaluated using exactly the same procedures employed to reduce (12). It is necessary in the course of the reduction to define an effective reciprocal lattice $\mathbf{G}^{\prime}$ for the overgrowth and, since $h$ is the potential overlap region common to the two crystals, we find, in analogy with (5),

$$
\mathbf{G}^{\prime} \equiv \mathbf{g}^{\prime}+2 \pi n \hat{\mathbf{n}} / 2 \hbar ; \quad(n=0, \pm 1, \ldots) . \quad \text {. . . . }
$$

The final result for the interaction energy per unit area, $E_{i}$, is :

$$
\begin{aligned}
E_{i}= & \left(\frac{N N^{\prime}}{A A^{\prime}}\right)^{1 / 2} \sum_{\mathbf{k}} v(\mathbf{k}) \exp (i \mathbf{k} \cdot \mathbf{B})\left\{\prod_{\mathbf{K}}+J_{0}\left(2 \mathbf{k} \cdot \mathbf{D}_{\mathbf{K}}\right) J_{\mathbf{0}}\left(2 \mathbf{k} \cdot \mathbf{C}_{\mathbf{K}}\right)\right\} \\
& \times\left\{\prod_{\mathbf{K}}^{+} J_{0}\left(2 \mathbf{k} \cdot \mathbf{D}_{\mathbf{K}}{ }^{\prime}\right) J_{\mathbf{0}}\left(2 \mathbf{k} \cdot \mathbf{C}_{\mathbf{K}}{ }^{\prime}\right)\right\}\left\{\delta_{\mathbf{k}, \mathbf{G}} \delta_{\mathbf{k}, \mathbf{G}^{\prime}}\right. \\
& +\sum_{n=1}^{\infty} \sum_{\mathbf{K}}[]_{1} \delta_{\mathbf{G}+n \mathbf{K}, \mathbf{G}^{\prime}} \delta_{\mathbf{k}, \mathbf{G}^{\prime}}+\sum_{n=1}^{\infty} \sum[]_{\mathbf{K}}\left[\delta_{1^{\prime}} \delta_{\mathbf{G}}+n \mathbf{K}, \mathbf{G}^{\prime} \delta_{\mathbf{k}, \mathbf{G}}\right. \\
& \times \sum_{n, n^{\prime}=1}^{\infty} \sum_{\mathbf{K}, \mathbf{K}^{\prime}}[]_{1}[]_{1^{\prime}} \delta_{\mathbf{G}+n \mathbf{K}+n^{\prime} \mathbf{K}^{\prime}, \mathbf{G}^{\prime}} \delta_{\mathbf{k}, \mathbf{G}+n \mathbf{K}} \\
& +\sum_{n, m=1}^{\infty} \sum_{\mathbf{K}, \mathbf{L}}[]_{2} \delta_{\mathbf{G}+n \mathbf{K}+m \mathbf{L}, \mathbf{G}^{\prime} \delta_{\mathbf{k}, \mathbf{G}^{\prime}}} \\
& \left.+\sum_{n, m=1}^{\infty} \sum_{\mathbf{K}, \mathbf{L}}[]_{2^{\prime}} \delta_{\mathbf{G}+n \mathbf{K}+m \mathbf{L}, \mathbf{G}^{\prime}} \delta_{\mathbf{k}, \mathbf{G}}+\ldots\right\}, \ldots . .
\end{aligned}
$$


where the brackets []$_{1}$ and []$_{2}$ refer to the corresponding bracketed expressions in (15) and the brackets []$_{1^{\prime}}$ and []$_{2^{\prime}}$ are the same expressions with $\mathbf{C}_{\mathbf{K}}$, $\mathbf{D}_{\mathbf{K}}$ replaced by $\mathbf{C}_{\mathbf{K}}{ }^{\prime}, \mathbf{D}_{\mathbf{K}}{ }^{\prime}$. The translations $\mathbf{C}_{0}$ and $\mathbf{C}_{0}{ }^{\prime}$ have been combined to a single vector $\mathbf{B}$.

Two things can immediately be seen from the form of $E_{i}$. Firstly, since, in the sums over $\mathbf{k}$ and $\mathbf{K}$, each term is accompanied by a term in - $\mathbf{k}$ and $-\mathrm{K}$, and since the coefficients obey (3) and (10), $E_{i}$ is a real quantity, as it must be physically. Secondly, the expression is symmetric in form with respect to contributions from overgrowth and substrate so that the same result would have been arrived at had we begun our calculation with crystal B instead of crystal A. This again is physically necessary.

Though the expression (19) is complicated, the number of terms involved is not actually large since the Kronecker deltas select just one term from each summation. A further restriction on possible distortion terms is imposed by the finite mesh size of each undistorted crystal lattice. The distortions $\mathbf{F}(\mathbf{R})$ and $\mathbb{F}^{\prime}\left(\mathbf{R}^{\prime}\right)$ are not really continuous functions but have meaning only at the lattice points. Thus, just as in the theory of phonons in crystals, of which these distortions are the static analogue, the 'wave vectors' $\mathbf{K}$ must lie within the first Brillouin zone of the crystal concerned, these Brillouin zones being defined by the surface reciprocal lattice vectors $\mathbf{g}$ and $\mathbf{g}^{\prime}$ respectively. We shall see in the next section how these restrictions can be automatically included in the formalism.

We may also anticipate a result from the next section and remark that there are, in general, six equations relating to the twelve components of the distortion vectors $\mathbf{C}_{\mathbf{K}}, \mathbf{D}_{\mathbf{K}}, \mathbf{C}_{\mathbf{K}}{ }^{\prime}$, and $\mathbf{D}_{\mathbf{K}}{ }^{\prime}$ associated with any distortion $\mathbf{K}$. This effectively reduces the number of independent distortions (which will form our set of variational parameters) by a factor 2.

Finally we note that if one crystal has an $n$-fold axis of rotation symmetry normal to the interface plane and the other an $m$-fold axis, then the number of independent distortion components is further reduced by the highest common factor of $n$ and $m$. These observations, combined with the fact that a physically reasonable potential has only a finite, and not very large, extension in $\mathbf{k}$-space, mean that the number of independent variational parameters in (19) is not unmanageably large. We shall see later that they can also be easily placed in order of importance.

\section{§ 4. ELASTIC ENERGY}

We now come to calculate the elastic strain energy associated with the distortion components $\mathbf{F}_{\mathbf{K}}$ and $\mathbf{F}_{\mathbf{K}}{ }^{\prime}$. These distortions have a maximum amplitude of about one interatomic spacing for modes of long wavelength (small $\mathbf{K}$ ) while the maximum amplitude for modes with $\mathbf{K}$ near the zone boundary cannot exceed a few tenths of one interatomic spacing and, except in the case of very soft crystals, will usually be much less than this. Typical total strains probably therefore do not exceed about $20 \%$ and are often much less than this, so that it is a reasonable approximation 
to calculate the strain energy on the assumption that each crystal behaves as an elastic continuum.

Even the case of an elastic continuum subject to periodic displacements at its free plane surface is a complicated problem, though its simpler aspects are well documented (Sokolnikoff 1956, van der Merwe 1963). Whilst there is only a moderate complication in treating crystals of finite thickness (van der Merwe 1963), we shall content ourselves with a discussion of the simpler problem posed by semi-infinite crystals and even here we shall seek the simplest approximation consistent with the needs of our problem.

We assume that each crystal can be treated as an isotropic elastic continuum whose moduli are the same for all distortions with wave vector K lying within its first Brillouin zone. Let us further suppose that we can define and measure an elastic modulus for each of the three types of distortion $\mathbf{F}_{\mathbf{K}(1)}$ (with $\mathbf{F}$ parallel to $\mathbf{K}$ ), $\mathbf{F}_{\mathbf{K}(2)}$ (with $\mathbf{F}$ perpendicular to $\mathbf{K}$ but still lying in the interface) and $\mathbf{F}_{\mathbf{K}(3)}$ (with $\mathbf{F}$ normal to the interface); and denote them by $\eta_{1}, \eta_{2}$ and $\eta_{3}$ respectively. This can be done for both substrate and overgrowth, giving in the latter case $\eta_{1}{ }^{\prime}, \eta_{2}{ }^{\prime}$ and $\eta_{3}{ }^{\prime}$. We shall also assume that the two crystals are of similar structure so that $\eta_{i} / \eta_{i}{ }^{\prime}$ is independent of $i$.

Now, since all stresses must balance across the interface, this simplified set of assumptions gives

$$
\eta_{i} \mathbf{F}_{\mathbf{K}^{(i)}}=\eta_{i}{ }^{\prime} \mathbf{F}_{\mathbf{K}(i)}^{\prime} \quad \text {. . . . . . . . . . . }
$$

for all $i$, provided $\mathbb{K}$ lies within the first Brillouin zone of each crystal. To ensure that distortions corresponding to larger $\mathbf{K}$ values vanish, we assume that $\eta_{i}=\infty$ when $\mathbf{K}$ lies outside the first Brillouin zone of $\mathbf{g}$, with a similar requirement for $\eta_{i}{ }^{\prime}$.

When superposition of different distortion components is considered we see that, whilst distortions of different $\mathbf{K}$ are orthogonal and can be freely superposed, cross-terms occur between the different $\mathbf{F}_{\mathbf{K}(i)}$ belonging to the same value of $\mathbf{K}$. For example $\mathbf{D}_{\mathbf{K}(1)}$, which is parallel to $\mathbf{K}$, gives rise to periodic regions of compression and tension which, through an effective 'surface Poisson's ratio', cause displacements normal to the interface and so contribute to $\mathbf{C}_{\mathbf{K}(3)}$. The effect is, however, not large and is compensated to a large extent by a displacement of the overgrowth in the same direction. The effect has been considered in greater detail by van der Merwe (1963) who concluded that its influence on total energy was small. In the interests of simplicity we shall therefore neglect such cross terms in the discussion which follows.

The elastic strains fall off exponentially away from the interface in each crystal and the elastic strain associated with a particular distortion can be calculated by integrating the product $\frac{1}{2}$ (stress) $\times$ (strain), throughout the volume. More simply, however, since we have already defined appropriate elastic moduli, we may integrate the same effective product over the interface to give, for unit area:

$$
\left.E_{\mathrm{e}}=\sum_{i} \sum_{\mathbf{K}}+K\left[\eta_{i}\left(C_{\mathbf{K}(i)^{2}}+D_{\mathbf{K}(i)^{2}}\right)^{2}+\eta_{i}{ }^{\prime}\left(C_{\mathbf{K}}^{\prime}(i)^{2}+D_{\mathbf{K}^{\prime}(i)^{2}}\right)^{2}\right)\right]
$$


where $K, C_{\mathbf{K}}$ and $D_{\mathbf{K}}$ are the moduli of $\mathbf{K}, \mathbf{C}_{\mathbf{K}}$ and $\mathbf{D}_{\mathbf{K}}$ respectively. This equation, together with (20) and the condition that $\eta_{i}$ and $\eta_{i}{ }^{\prime}$ become infinite outside their respective first Brillouin zone, sums up our treatment of the elastic energy.

\section{$\S 5$. Discussion}

The energy of the interface in its state of arbitrary general strain, relative to the sum of the surface energies of the two separated crystals as a zero, is found by summing terms corresponding to the interaction energy across the interface and the elastic strain energy in the two crystals:

$$
E=E_{i}+E_{\mathrm{e}}
$$

where $E_{i}$ and $E_{\mathrm{e}}$ are defined by (19) and (21) respectively and the coefficients involved are related by (20). The energy given by (22) contains a large set of vectorial variational parameters $\mathbf{C}_{\mathbf{K}}, \mathbf{C}_{\mathbf{K}}{ }^{\prime}, \mathbf{D}_{\mathbf{K}}$ and $\mathbf{D}_{\mathbf{K}}{ }^{\prime}$ representing all possible relevant distortions of the interface, and the true value of $E$ is found by minimizing (22) with respect to these parameters. The whole problem is now best treated numerically and indeed we shall discuss the results of a typical ealculation in a subsequent paper. It is worth while, however, to make a few observations on the general form of the expression for $E$ and on its relation to the dislocation model.

In the first place we note from (19) that minima occur in $E$ for lattice ratios and orientations for which $\delta_{\mathbf{G}, \mathbf{G}^{\prime}}=\delta_{\mathbf{g}, \mathbf{g}^{\prime}}=1$ and that the depth of a minimum is proportional to the $v(\mathbf{G})$ involved. This coincidence of reciprocal lattice vectors between the surfaces of overgrowth and substrate implies a corresponding coincidence between certain atomic positions in the surfaces of the two crystals, the number of such atomic coincidences being greatest for small values of $\mathbf{g}$ and $\mathbf{g}^{\prime}$. Generally the potential components $v(\mathbf{G})$ will also be largest for small values of $\mathbf{G}$ so that we can assert that significant minima occur in the interfacial energy whenever the symmetry, orientation and lattice parameters of the two crystals involved are such that there are a large number of atomic coincidences across the interface plane.

Figure $1(a)$ shows an example of such a general matching between (100) faces of two face-centred cubic crystals with lattice parameters in the ratio $\sqrt{ } 2: 1$ and relative orientation $45^{\circ}$. Depending upon the form of $v(r)$, the configuration of minimum energy may be related to the 'coincident' one by a simple translation, as shown in fig. $1(b)$. Much more general cases than this are, of course, possible.

When we are close to such a matched condition, as shown for a particular. case in fig. 2 , which is drawn in reciprocal space, then one of the distortion vectors $\mathbf{K}$ (together with those grouped with it by symmetry) will be small and most other $\mathbf{K}$-vectors will be large. We can show that distortions based upon this set of shortest $\mathbf{K}$-vectors play a dominant part in determining the energy. To see this, suppose that all crystal distortions are small so that all the Bessel functions in (19) can be replaced by the first term 
Fig. 1

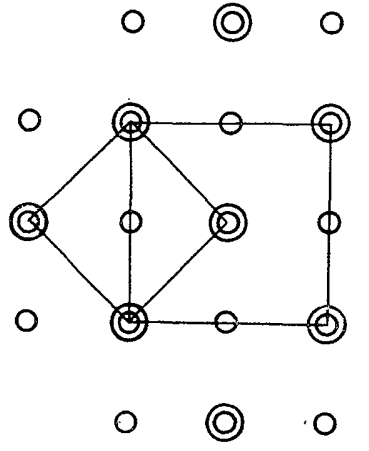

(a)

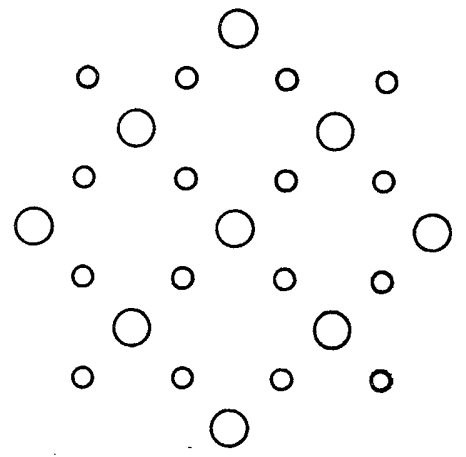

(b)

(a) An example of generalized matching between (100) faces of two f.c.c. crystals with lattice parameters in the ratio $\sqrt{ } 2: 1$ and relative orientation $45^{\circ}$. (b) For certain potentials the configuration of minimum energy may differ from the 'matched' configuration by' a simple relative translation between the two lattices.

Fig. 2<smiles>[O]</smiles>

$\mathrm{K}_{2}$<smiles>[Os]</smiles><smiles>O[Hg]</smiles><smiles>[O][O]</smiles><smiles>[Ca]</smiles><smiles>O</smiles><smiles>[Co]</smiles><smiles>[O]</smiles>

Portion of the reciprocal lattices, near the origin, of two similar crystals related by a twist boundary of small angle. Principal distortion K-vectors are shown joining reciprocal lattice points of the two sets.

in their series expansion, and consider the contribution to the energy from a distortion pair $\mathbf{D}_{\mathbf{k}}, \mathbf{D}_{\mathbf{k}}{ }^{\prime}$. This contribution is of the general form:

$$
E \approx \alpha v(\mathbf{G}) \cos (\mathbf{G} . \mathbf{B}) \mathbf{G} .\left(\mathbf{D}_{\mathbf{K}}+\mathbf{D}_{\mathbf{K}}{ }^{\prime}\right)+\beta K\left(\mathbf{D}_{\mathbf{K}}+\mathbf{D}_{\mathbf{K}}{ }^{\prime}\right)^{2}
$$

where $\alpha$ and $\beta$ are positive constants. The displacement $\mathbf{B}$ can be chosen so that $v(\mathbf{G}) \cos (\mathbf{G} . \mathbf{B})$ is negative and variation of $\mathbf{D}_{\mathbf{K}}, \mathbf{D}_{\mathbf{K}}{ }^{\prime}$ (using $(20)$ ) to minimize $E$ leads to the results :

$$
\mathbf{D}_{\mathbf{K}} \propto \mathbf{D}_{\mathbf{K}}{ }^{\prime} \propto K^{-1} ; \quad E \propto-|v(\mathbf{G})|^{2} K^{-1}, \quad . \quad . \quad . \quad .
$$

which show the dominant role of distortions of small $\mathbf{K}$. These results 
clearly become invalid as $\mathbf{K} \rightarrow 0$, but they do imply that the minimum at the condition of generalized matching between the two lattices forms the apex of a sharp cusp.

Such conditions of near matching are of particular interest because it is just these cases which can be validly treated by a dislocation model such as that of van der Merwe. The elastic distortion associated with a series of widely spaced dislocations in one dimension is of saw-tooth shape which has Fourier components of the form $\mathbb{D}_{n \mathbf{K}} \approx(-1)^{n} n^{-1} \mathbf{D}_{\mathbf{K}}$. To compare this model with our present treatment we must look at the role of distortion components of the form $\mathbb{D}_{n \mathbf{K}}$, where $\mathbf{K}$ is the smallest distortion wave vector as discussed above. Those distortions with $n>1$ enter through terms like $J_{1}\left(2 \mathbf{G}_{0} \cdot \mathbf{D}_{2 \mathbf{K}}\right) J_{1}\left(2 \mathbf{G}_{0} \cdot \mathbf{D}_{-\mathbf{K}}\right)$ associated with the potential component $v\left(\mathbf{G}_{0}\right)$, or through $J_{1}\left(4 \mathbf{G}_{0} \cdot \mathbf{D}_{2 \mathbf{K}}\right)$ associated with $v\left(2 \mathbf{G}_{0}\right)$, etc. It is clear that these higher terms will contribute less to the total energy than does the term with $n=i$ because their strain energy for a given amplitude is higher by a factor $n$. We therefore expect $\mathbf{D}_{n \mathbf{K}}$ to decrease with increasing $n$, as required by the validity of the dislocation model, and to vanish when $n \mathbf{K}$ passes outside the first Brillouin zone.

It is clear that, in its range of validity near the cusped energy minimum corresponding to $1: 1$ matching between substrate and overgrowth, the the dislocation model may provide a more convenient means of discussing the problem than does the present technique. When, however, $\mathbf{K}$ is an appreciable fraction of the distance to the boundary of the first Brillouin zone, the dislocation model is no longer appropriate and,' as usually formulated, it will average out much of the ine structure associated with higher-order matching conditions.

It should also be pointed out that the present treatment automatically includes all the symmetry elements of the crystals involved instead of requiring an artificial symmetrization at the end of the calculation, as is often the case with dislocation models. This is of particular importance in the case of a twist boundary between two similar crystals, as shown in fig. 2. It has often been the practice in dislocation models to treat such a boundary by a superposition of two sets of dislocations with mutually perpendicular $\mathbf{K}$ values to give a dislocation net. Two such sets do indeed exist, corresponding to $\mathbf{K}_{\mathbf{1}}$ and $\mathbf{K}_{\mathbf{2}}$ in the figure, but their superposition will not give a complete solution to the problem if there are potential components at reciprocal lattice points other than those with coordinates $(l, 0)$ or $(0, m)$ respectively. Consider, for example, the point $(1,1)$. If this superposition approximation is used, then its potential $v\left(G_{11}\right)$ enters only through terms like $J_{1}\left(2 \mathbf{G}_{11} \cdot \mathbf{D}_{\mathbf{k} 1}\right) J_{1}\left(2 \mathbf{G}_{11} \cdot \mathbf{D}_{\mathbf{K} 2}\right)$, which is of second order, instead of through the first-order term $J_{1}\left(2 \mathbf{G}_{11} \cdot \mathbf{D}_{\mathbf{K} 3}\right)$. The present treatment takes proper acount of these terms.

\section{§ 6. CONCLUSION}

We have presented the formal theory of a method for the calculation of the energy of a wide class of crystal interfaces, which gives promise of 
realistic energy calculations without undue labour or computing time. The formal exposition of the theory already exhibits several important features associated with the interfacial energy, such as the cusped energy minima near conditions of generalized atomic matching across the interface, and its relationship to the dislocation model can be seen in the region where both approaches are valid. The present technique is, however, of more general validity than an ordinary dislocation model and, being a variational procedure, is also well adapted to first order calculations where a minimization with respect to a few dominant parameters will yield both an upper bound and a reasonable approximation to the interfacial energy. Some of these points will be pursued in a subsequent publication.

\section{ACKNOWLEDGMENT}

Work on this subject was begun jointly by the two authors and the theory was brought to its present form by one of us (N.H.F.) during tenure of a Nuffield Fellowship at Bristol University.

\section{RFFERENCES}

Amelinckx, S., and Dekeyser, W., 1959, Solid State Physics, Vol. 8, edited by F. Seitz and D. Turnbull (New York: Academic Press), p. 327.

Brooks, H., 1952, Metal Interfaces (Cleveland, Ohio: American Society for Metals), pp. 20-64.

Du Plessis, J. C., and van der Merwe, J. H., 1965, Phil. Mag., 11, 43.

FLETCHER, N. H., 1964, J. appl. Phys., 35, 234, 2276.

Frank, F. C., and VAN DeR Merwe, J. H., 1949, Proc. roy. Soc. A, 198, 216.

Read, W. T., 1963, Dislocations in Crystals (New York: MoGraw-Hill), Chap. $11-13$.

READ, W. T., and SHOCKLEY, W., 1950, Phys. Rev., 78, 275.

SokoLnIKorF, I. S., 1956, Mathematical Theory of Elasticity (New York: MoGraw-Hill), pp. 328-343, 354-358.

van Der Merwe, J. H., 1950, Proc. phys. Soc., Lond. A, 63, 616; 1963, J. appl. Phys., 34, 117, 123, 2420 .

Watson, G. N., 1944, Theory of Bessel Functions (Cambridge University Press), p. 14. 